\title{
PERCEPÇÃO SOBRE ASPECTOS DA ACESSIBILIDADE DA PESSOA COM DEFICIÊNCIA FÍSICA ADQUIRIDA, RESIDENTE NO MUNICÍPIO DE ITAJAÍ/SC
}

\author{
PERCEPTION ON ASPECTS OF THE PERSON WITH DISABILITIES \\ ACCESSIBILITY PHYSICAL ACQUIRED, RESIDENT IN MUNICIPALITY OF ITAJAÍ / \\ SC
}

\author{
Francielly Nalin ${ }^{1}$ \\ Vanessa G Groh ${ }^{2}$ \\ Fabiola Hermes Chesani ${ }^{3}$ \\ Tatiana Mezadri ${ }^{4}$ \\ Léo Lynce Valle de Lacerda ${ }^{5}$
}

Resumo: A acessibilidade arquitetônica é a possibilidade e a condição de alcance para que a pessoa com deficiência física utilize espaços de uso coletivo e meios físicos de uma forma mais autônoma. Este estudo objetivou analisar a percepção sobre aspectos da acessibilidade da pessoa com deficiência física adquirida residente na cidade de Itajaí/SC. Participaram da pesquisa 164 pessoas com deficiência física adquirida que pertenciam a cinco Unidades Básicas de Saúde de Itajaí e as redes de apoio (Clínica de fisioterapia, Centro Especializado em Reabilitação II, Associação de Pais e Amigos dos Excepcionais e Associação dos Deficientes Físicos da Foz do Rio Itajaí). Os dados foram coletados por meio de um formulário com questões sobre aspectos da acessibilidade. Foi realizada análise estatística descritiva para sistematização dos dados. Os resultados demonstraram que a percepção do deficiente físico, com relação à acessibilidade, é ampla; dos quais 60\% apontaram a falta de estrutura arquitetônica satisfatória em seu domicílio e 56,09\% observaram a mesma condição em ambientes de uso coletivo. Esses resultados demonstram que a pessoa com deficiência física adquirida reconhece as barreiras arquitetônicas que dificultam e, muitas vezes, impossibilitam sua locomoção e convivência social, impedindo sua autonomia de ir e vir para onde desejar.

Palavras-chave: Estruturas de acesso; barreiras arquitetônicas; pessoa com deficiência física.

Abstract: The architectural accessibility is the possibility and scope of condition for the physically disabled person to use spaces for collective use and physical means of a more autonomous way. This study aimed to analyze the perception of some aspects of accessibility for people with disabilities resident of the city of ItajailSC. Therefore the methodology adopted was the simple and quantitative descriptive. The participants were people with acquired disabilities belonging to the five Basic Health Units of Itajai and support networks such as physiotherapy clinic, the Specialized Center for Rehabilitation II, the Association of Parents and Friends of Exceptional Children and the Association Handicap of Foz do Rio Itajaí. Data were collected through a questionnaire with questions about some aspects of accessibility. The sample of 164 people with acquired physical disability, the collected data were statistically analyzed. In the analysis of the results it can be noted that the perception of the disabled person in relation to accessibility is broad but lacking architectural structure that satisfies both his home and in public use, so we found that about $60 \%$ of disabled people reported not have accessibility in his home, and $56.09 \%$ reported not having accessibility collective use. Consider that the perception of handicapped there are still many architectural

\footnotetext{
1 Universidade do Vale do Itajaí, Univali, Brasil. E-mail: francinalin@hotmail.com.

2 Universidade do Vale do Itajaí, Univali, Brasil. E-mail: vanessa.groh@hotmail.com.

3 Universidade do Vale do Itajaí, Univali, Brasil. E-mail: fabiola.chesani@univali.br.

4 Universidade do Vale do Itajaí, Univali, Brasil. E-mail: mezadri@univali.br.

5 Universidade do Vale do Itajaí, Univali, Brasil. E-mail: leolynce@univali.br.
} 
barriers that do not favor them, making it impossible to locomotor performance preventing the person from having a range of come and go where you want.

Keywords: Access structures; architectural barrier; physical disability.

\section{INTRODUÇÃO}

A deficiência e a incapacidade não são apenas uma consequência das condições de saúde/doença, mas são determinadas, também, pelo contexto do meio ambiente físico e social, pelas diferentes percepções culturais e atitudes em relação à deficiência, pela disponibilidade de serviços e de legislação. Sabe-se que as pessoas com deficiência física muitas vezes encontram dificuldades na sua trajetória de vida, deparando-se com barreiras de ordens ambientais e sociais, as quais foram apontadas, pela primeira vez, na Classificação Internacional de Funcionalidade, Incapacidade e Saúde (CIF), que descreve os termos funcionalidade e incapacidades relacionadas às condições de saúde da pessoa. $\mathrm{O}$ termo funcionalidade se refere aos componentes de funções e estruturas do corpo, atividade e participação social. $O$ aspecto positivo da CIF é a funcionalidade e aspecto negativo são as incapacidades (FARIAS; BUCHALLA, 2005).

Diante desses fatos, e de acordo com a Secretaria Nacional de Promoção dos Direitos da Pessoa com Deficiência, a acessibilidade pode ser definida como um atributo essencial para o ambiente, o que garante às pessoas com deficiência física uma melhoria da qualidade de vida. Essa acessibilidade precisa estar presente no meio físico, nos espaços, no transporte, na informação e comunicação, comparecendo tanto na cidade como no campo, nos serviços e instalações abertos ao público ou de uso público e nos sistemas e tecnologias da informação e comunicação.

Conforme a Associação Brasileira de Normas Técnicas (2004, p.2), a acessibilidade significa "possibilitar a condição de alcance, percepção e entendimento para a utilização com segurança e autonomia de edificações, espaço, mobiliário, equipamento urbano e elementos". No entanto, ainda que existam normas, legislações e investimentos para a remoção das barreiras, o que se observa é uma aplicação de forma bem restrita, e o que se percebe é uma falta de acessibilidade no ambiente em geral (MACHADO; LIMA, 2015).

Nesse sentido, esta pesquisa objetivou analisar a percepção sobre aspectos da acessibilidade no ambiente domiciliar e coletivo da pessoa com deficiência física adquirida, residente na cidade de Itajaí/SC, buscando fornecer subsídios que contribuam com as ações governamentais para melhorias no acesso dessas pessoas a um convívio social igualitário. 


\section{MÉTODO}

Neste trabalho, foi realizou-se uma pesquisa transversal de abordagem quantitativa, envolvendo pessoas com deficiência física adquirida de ambos os sexos, residentes no município de Itajaí/SC.

Os dados foram coletados em pontos de atenção à saúde, sendo: cinco Unidades Básicas de Saúde (que foram selecionadas por facilidade de acesso dos pesquisadores), a Clínica de Fisioterapia da Universidade do Vale do Itajaí, o Centro Especializado em Reabilitação (CER II) do município de Itajaí, bem como, nas redes de apoio à pessoa com deficiência física (a Associação dos Deficientes Físicos da Foz do Rio Itajaí e a Associação de Pais e Amigos dos Excepcionais - APAE).

A partir das equipes da Estratégia Saúde da Família das unidades registradas, as pessoas com deficiência física adquirida foram identificadas e entrevistadas em suas residências com data e horário previamente agendados. As que frequentavam o CER II e a clínica de fisioterapia foram entrevistadas no próprio local, em dias e horários previamente agendados. As pessoas que frequentavam as redes de apoio, que serviram de ponto de coleta para o estudo, foram entrevistadas nas sedes das associações. Os inquéritos foram realizados por acadêmicos, previamente treinados, dos cursos de Nutrição e Fisioterapia de uma Universidade do Vale do Itajaí, no período de fevereiro de 2015 à fevereiro de 2016.

Os critérios de inclusão estabelecidos foram: ter deficiência física adquirida (paraplegia, paraparesia, monoplegia, monoparesia, tetraplegia, tetraparesia, triplegia, triparesia, hemiplegia, hemiparesia, amputação ou ausência de membro, membros com deformidade adquirida e esclerose amiotrófica).

O instrumento de coleta de dados foi um questionário aplicado aos participantes da pesquisa, individualmente, e em locais combinados com os participantes. As questões que nortearam o questionário foram sobre as seguintes variáveis: idade; sexo; tipo de deficiência, ocupação e os itens acessíveis no ambito domiciliar e coletivo.

No questionário o participante marcava os itens acessíveis presentes nos espaços de uso domiciliar e coletivo. Os itens marcados de uso domiciliar das pessoas com deficiência física se referiram a: almofadas especiais, banheira adaptada, barra de apoio nos corredores,barra de apoio para vaso sanitário, barra de apoio no chuveiro, cama adaptada, campanhias para alarmes, carro adapatdo, chuveiro adaptado, corredores alargados, elevadores/plataformas, portas alargadas, rampas de acesso, tapetes antiderrapantes, torneiras adaptadas, vaso adpatado, escadas com corrimão, escadas de acesso interno, piso tátil. Quanto os aspectos acessíveis marcados pelos participantes de uso coletivo foram os seguintes: barra de apoio corredores, barra de apoio no chuveiro, barra de apoio no vaso, calçadas adaptadas, corredores alargados, elevadores/plataformas, portas alargadas, rampas 
de acesso, semáforos adaptados, torneiras adaptadas, transporte público adaptado, vaga de estacionamento reservadas, vaso adaptado, piso escorregadio, piso tátil e escadas com corrimão. Estes itens de acessibilidade domiciliar e coletivo seguiram alguns parâmetros da ABNT NBR 9050.

A análise da acessibilidade foi realizada em duas fases: na primeira computouse o percentual de itens marcados de acessibilidade, com base no número de marcações que cada respondente efetuou nos itens respectivos. Do total de itens marcados para cada respondente foram computados as quantidades relativas a equipamentos de acessibilidade e calculadas os respectivos percentuais. Na segunda fase foram examinados aqueles equipamentos de maior frequência de marcação.

As comparações dos valores médios da idade, tempo de deficiência e percentuais de equipamentos de acessibilidade nos domicílios e coletivos de acordo com o sexo e as características socioeconômicas e tipo de deficiência fisica, foram realizadas conforme o número de níveis apresentado pelo fator categórico: para sexo foi utilizado o teste-t; para o tipo de ocupação, o tipo de deficiência e a renda atual foi utilizada a análise de variância para um fator seguido do Teste de Tukey, quando necessário. Posteriormente foram examinadas as relações entre a idade, o tempo de deficiência e os percentuais de equipamentos nos domicílios e coletivos por meio do índice de correlação linear de Pearson. Optou-se pelos testes paramétricos tendo em vista a distribuição normal dos dados. O nível de significância adotado foi de $5 \%$. aplicativo Statistica v.10 (STATSOFT, 2011) foi utilizado em todas as análises e nas representações gráficas dos resultados.

Esta pesquisa foi aprovada pelo Comitê de Ética em Pesquisa da Universidade do Vale do Itajaí com protocolo № 694.257, financiada pela Fundação de Amparo à Pesquisa de Santa Catarina (FAPESC - Programa Universal), pelo Fundo de Apoio à Manutenção e ao Desenvolvimento da Educaçao Superior (FUMDES- artigo 171) e Artigo 170.

\section{RESULTADOS}

Foram analisados dados de 164 indivíduos com deficiência física adquirida residentes no município de Itajaí/SC. As características sociodemográficas e de saúde estão descritas na Tabela 1, a maior faixa etária foi encontrada foi acima de 40 anos $(76,3 \%)$, a variável sexo obteve percentuais semelhantes $(49,4 \%$ eram mulheres e $50,6 \%$ homens) e a maioria referiu ser branco $(76,2 \%)$.

Quando analisados os itens acessíveis presentes no domicílio pelas pessoas com deficiência física, constatou-se que o item "rampas de acesso" foi o mais marcado $(26,83 \%)$ seguido de tapetes antiderrapantes $(18,9 \%)$ e "barras de apoio para vaso sanitário e no chuveiro" e "portas alargadas" com $17 \%$ de marcações, no entanto, 
"almofadas" e "camas especiais" foram apontadas com 3,05\% do total de respondentes como os itens menos acessíveis no domicílio (Tabela 2).

Tabela 1 - Características sociodemográficas e de saúde das pessoas com deficiência física adquirida do município de Itajaí (SC). Fevereiro de 2015 a 2016.

\begin{tabular}{|c|c|c|}
\hline Variável & $\mathbf{N}$ & $\%$ \\
\hline \multicolumn{3}{|l|}{ Sexo } \\
\hline Feminino & 81 & 49,4 \\
\hline Masculino & 83 & 50,6 \\
\hline \multicolumn{3}{|l|}{ Faixa Etária (anos) } \\
\hline $10-19$ & 07 & 4,2 \\
\hline $20-39$ & 32 & 19,5 \\
\hline $40-59$ & 58 & 35,3 \\
\hline 60 e mais & 67 & 41 \\
\hline \multicolumn{3}{|c|}{ Tipos de deficiência física } \\
\hline Plegias & 95 & 57,9 \\
\hline Paresias & 43 & 26,2 \\
\hline Amputações & 26 & 15,8 \\
\hline \multicolumn{3}{|l|}{ Ocupação } \\
\hline Desempregado & 14 & 9,4 \\
\hline Do lar & 28 & 18,8 \\
\hline Aposentado & 55 & 36,9 \\
\hline Benefício por invalidez & 38 & 25,5 \\
\hline Assalariado & 14 & 9,4 \\
\hline \multicolumn{3}{|l|}{ Renda* } \\
\hline Até 2 SM & 99 & 60,4 \\
\hline$>2$ a 4 SM & 50 & 30,5 \\
\hline$>4 \mathrm{SM}$ & 15 & 9,1 \\
\hline
\end{tabular}

Tabela 2 - Porcentagem de itens de acessibilidade das pessoas com deficiência física adquirida residentes em Itajaí/SC, marcados em uso domiciliar.

\begin{tabular}{lrr}
\hline ITEM EM DOMICILIO & № & \% \\
\hline Adaptados & 5 & 3,0 \\
Almofadas especiais & 2 & 1,2 \\
Banheira & 14 & 8,5 \\
Barra de apoio corredores & 28 & 17,0 \\
Barra de apoio para vaso sanitário & 28 & 17,0 \\
Barras de apoio no chuveiro & 5 & 3,0 \\
Cama especial & 11 & 6,7 \\
Campainhas para alarmes & 18 & 10,9 \\
Carro adaptado & 10 & 6,1 \\
Chuveiro adaptado & 20 & 12,2 \\
Corredores alargados & 6 & 3,6 \\
Elevadores/plataformas & 29 & 17,6 \\
Portas alargadas & 44 & 26,8 \\
Rampas de acesso & 31 & 18,9 \\
Tapetes antiderrapantes & 11 & 6,7 \\
Torneiras adaptadas & 13 & 7,9
\end{tabular}


Escadas com corrimão

10

20

6,1

Escadas de acesso interno

Piso tátil

Não responderam

Quando analisados os itens acessíveis em uso coletivo pelas pessoas com deficiência física, constatou-se que "rampas de acesso" foi o mais marcado $(35,4 \%)$ seguido de vaga de estacionamento reservada (32,9\%), transporte público adaptado $(26,2 \%)$ no entanto, "barras de apoio no chuveiro" e "torneiras adaptadas" foram apontadas com $6,1 \%$ do total de respondentes como os itens menos acessíveis (Tabela 3).

Tabela 3 - Porcentagem de itens de acessibilidade das pessoas com deficiência física adquirida residentes em Itajaí/SC, marcados em uso coletivo.

\begin{tabular}{lcc}
\hline ITENS NO COLETIVO & № & $\%$ \\
\hline Adaptado & 42 & 25,6 \\
Barra de apoio corredores & 10 & 6,1 \\
Barra de apoio no chuveiro & 29 & 17,7 \\
Barra de apoio no vaso & 34 & 20,7 \\
Calçadas adaptadas & 40 & 24,4 \\
Corredores alargados & 12 & 7,3 \\
Elevadores/plataformas & 31 & 18,9 \\
Portas alargadas & 58 & 35,4 \\
Rampas de acesso & 39 & 23,8 \\
Semáforos adaptados & 10 & 6,1 \\
Torneiras adaptadas & 43 & 26,2 \\
Transporte público adaptado & 54 & 32,9 \\
Vaga de estacionamento reservadas & 24 & 14,6 \\
Vaso adaptado & 32 & 19,5 \\
Piso escorregadio & 24 & 14,6 \\
Piso tátil & 20 & 12,2 \\
Escadas com corrimão & 10 & 6,1 \\
Não responderam & & \\
\hline
\end{tabular}

A Tabela 4 demonstra as diferenças no percentual de equipamentos acessíveis em domicílio de acordo com as variáveis socioeconômicas e tipo de deficiência dos respondentes. Observa-se diferença significativa para as variáveis renda. Ou seja, quem recebe até dois salários mínimos possuem menos equipamentos acessíveis no domicílio do que aqueles que ganham mais de quatro salários. 
Tabela 4 - Percentual de equipamentos acessíveis em domicilio de acordo com as variáveis socioeconômicas e tipo de deficiência das pessoas com deficiência física adquirida, no município de Itajaí, de fevereiro de 2015 à 2016.

\begin{tabular}{|c|c|c|c|}
\hline \multirow{2}{*}{ VARIÁVEL } & \multicolumn{3}{|c|}{ PERCENTUAL DE EQUIPAMENTO NO DOMICÍLIO } \\
\hline & MD (IC 95\%) & $\mathbf{N}$ & VALOR DE $p^{*}$ \\
\hline \multicolumn{4}{|l|}{ Sexo } \\
\hline Masculino & $34,7(27,2-42,2)$ & 76 & \multirow{2}{*}{0,8039} \\
\hline Feminino & $36,0(28,7-43,5)$ & 73 & \\
\hline \multicolumn{4}{|l|}{ Faixa Etária } \\
\hline $10-19$ & $42,5(4,9-80,0)$ & 5 & \multirow{4}{*}{0,7215} \\
\hline $20-39$ & $34,8(21,6-47,9)$ & 28 & \\
\hline $40-59$ & $31,8(23,1-40,6)$ & 53 & \\
\hline 60 e mais & $38,0(30,0-46,1)$ & 63 & \\
\hline \multicolumn{4}{|l|}{ Ocupação } \\
\hline Desempregado & $33,6(15,0-52,3)$ & 14 & \multirow{5}{*}{0,3778} \\
\hline Do lar & $37,1(24,2-50,0)$ & 28 & \\
\hline Aposentado & $39,0(30,0-48,1)$ & 55 & \\
\hline Benefício por invalidez & $26,8(18,2-35,3)$ & 38 & \\
\hline Assalariado & $42,3(20,2-64,3)$ & 14 & \\
\hline \multicolumn{4}{|l|}{ Renda ** } \\
\hline Até 2 SM & $31,5^{\mathrm{a}},(25,0-38,0)$ & 95 & \multirow{3}{*}{0,0471} \\
\hline$>2$ a 4 SM & $38,7^{\mathrm{a} b}(29,0-48,3)$ & 42 & \\
\hline$>4 \mathrm{SM}$ & $53,0^{b}(36,4-69,6)$ & 14 & \\
\hline \multicolumn{4}{|l|}{ Tipo de deficiência } \\
\hline Plegias & $35,5(28,7-42,4)$ & 85 & \multirow{3}{*}{0,1315} \\
\hline Paresias & $41,6(30,7-52,5)$ & 39 & \\
\hline Amputação & $25,2(13,0-37,3)$ & 25 & \\
\hline
\end{tabular}

${ }^{*}$ Teste-t ao nível de $5 \%$ para as variáveis de sexo. Para as demais, Anova monofatorial ao nível de $5 \%$.

**Letras iguais sobrescritas após as médias denotam médias iguais no teste a posterior de Tukey.

A variável renda também apresentou diferença significativa ( $p=0,0471)$ : os que declararam ganhar até dois salários mínimos marcaram 31,5\% de itens acessíveis, e os que ganhavam acima de quatro salários mínimos marcaram um percentual de $53,0 \%$ de itens de acessibilidade em domicilio. Os respondentes que declararam ganhar de dois até quatro salários mínimos marcaram percentual intermediário $(38,7 \%)$ de itens.

Quando examinadas as relações entre gênero e tempo de deficiência encontrouse uma fraca relação negativa $(-33,4 \%)$, mas significativa $(p=0,0101)$ entre a idade e 0 tempo de deficiência nas mulheres, conforme o Gráfico 1. 
Gráfico 1 - Relação entre a idade e o tempo de deficiência das mulheres e homens com deficiência física adquirida em Itajaí/SC.

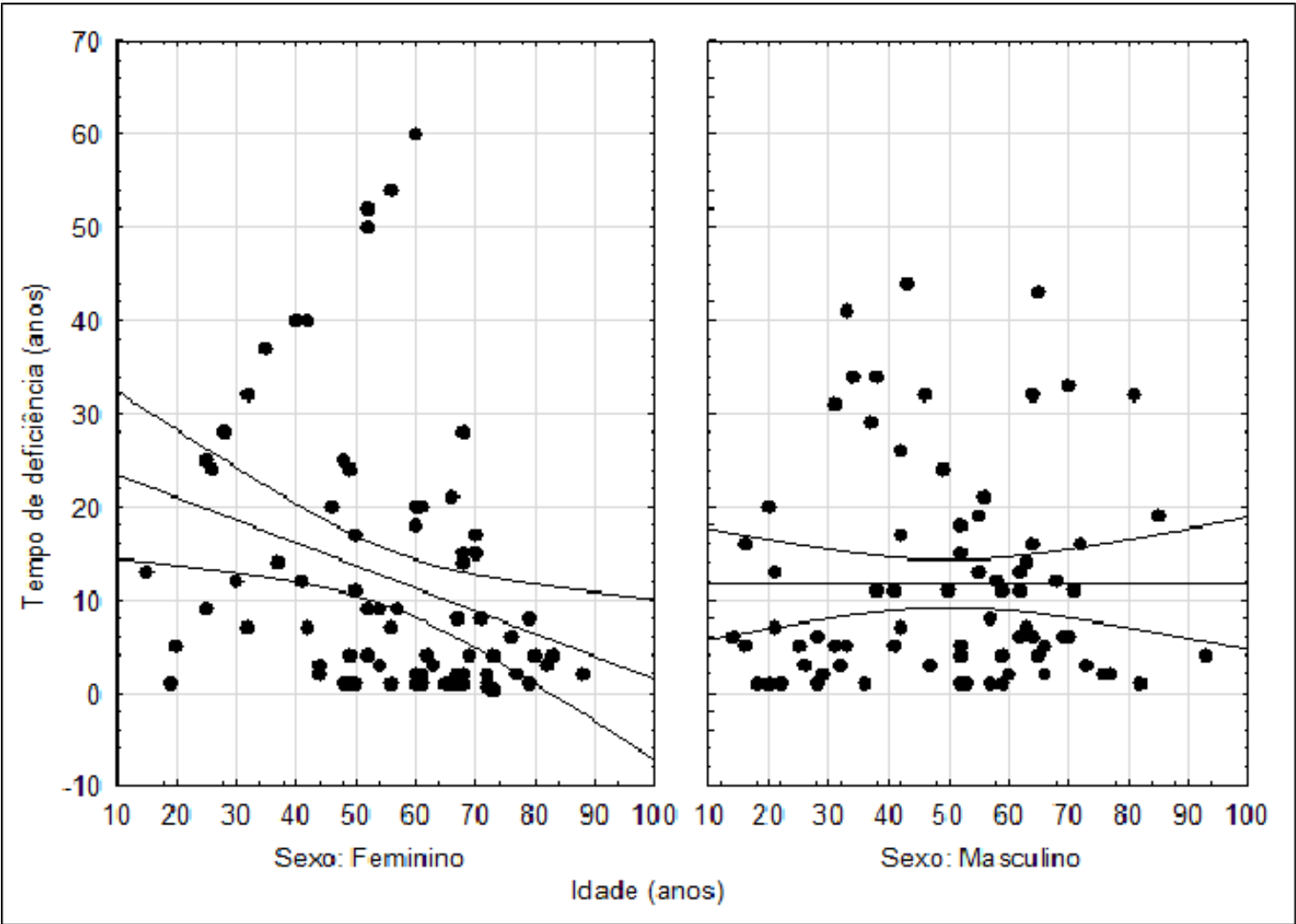

Não foram encontradas relações significativas entre a idade e percentual de equipamentos no domicílio ou a percepção no coletivo ou o tempo de deficiência e as demais variáveis.

\section{DISCUSSÃO}

A amostra avaliada no presente estudo apresentou percentuais semelhantes. De acordo com o último relatório do Censo Demográfico do IBGE (2013), no Brasil, a prevalência de deficiência física é maior em mulheres (com percentual de 8,6\%) com relação aos homens (5,3\%). Entretanto, em estudo realizado por Castro et al. (2008) com pessoas que adquiriram deficiência física no Estado de São Paulo, pode-se observar prevalência do sexo masculino sobre o sexo feminino. Algumas explicações para esses números se dão por fatores ligados às categorias profissionais dos homens, bem como maior exposição a fatores de risco como violência, álcool e trânsito (LEYTON et al., 2005; CASTRO et al, 2008; SCHOELLER et al, 2015).

A variável renda apresentou diferença significativa $(p=0,0471)$ : os que declararam ganhar até dois salários mínimos marcaram 31,5\% de itens acessíveis, e os que ganhavam acima de quatro salários mínimos marcaram um percentual de $53,0 \%$ de itens de acessibilidade em domicílio. Os respondentes que declararam 
ganhar de dois até quatro salários mínimos marcaram percentual intermediário $(38,7 \%)$ de itens.

Para Elias; Monteiro; Chaves (2008) existe grande relação entre incapacidade de pessoas com deficiência física e pobreza, uma vez que $80 \%$ destes indivíduos vivem com baixa renda. No estudo de Gasparoto; Alpino (2012), notou-se que as famílias tinham baixo poder aquisitivo, dificultando assim a questão acessibilidade em domicílio.

Com relação aos tipos de deficiências físicas encontradas na pesquisa, a plegia foi a mais citada com 57,9\% (95 marcações), seguida por paresia 26,2\% (43 marcações) e amputações com 15,8\% (26 marcações). Neste sentido, pode-se citar Nogueira (2014), que ao analisar o perfil epidemiológico da pessoa com deficiência física residente em Florianópolis, encontrou também maior índice de deficientes físicos com paraplegia, e relatou que essas causas predominantes das deficiências físicas são em decorrência dos acidentes de trânsito e das doenças crônicas não transmissíveis.

Já Schoeller et al. (2015), em estudo que buscou caracterizar as pessoas com lesão medular atendidas em um centro de reabilitação de referência, obteve maior percentual de pacientes com tetraplegia com relação a paraplegia.

Com relação aos itens acessíveis em domicilio marcados pelas pessoas com deficiência física, o que prevaleceu foram rampas de acesso foi o mais marcado seguido de tapetes antiderrapantes (18,9\%) e barras de apoio para vaso sanitário e no chuveiro e portas alargadas com $17 \%$ de marcações. No entanto, banheiras, almofadas e camas especiais foram apontadas com 3,05\% do total de respondentes como os itens menos acessíveis no domicílio.

Pode-se observar que no presente estudo a existência de rampas de acesso está presente na vida de $26,83 \%$ dos participantes, isto reflete que $73.17 \%$ dos participantes não tem de rampas de acesso no domicílio. Gasparotto; Alpino (2012) encontraram barreiras arquitetônicas ao avaliarem a acessibilidade domiciliar de crianças com deficiência física, pois identificaram que havia desníveis em cômodos e pátio da casa, dificultando seu acesso. Além disso, o número de rampas existentes era insuficiente e destas, nenhuma apresentava inclinação adequada.

Garanhani, Alves e Fujisawa (2009) avaliando a acessibilidade domiciliar de pessoas após episódio de Acidente Vascular Encefálico, observaram que o acesso da maioria dos domicílios visitados possuía degraus e terrenos irregulares; tornando-se, portanto, obstáculos para estas pessoas. Para Pynoos e Overton (2003), as áreas da casa que apresentam maiores riscos são o acesso a casa, as escadas e o banheiro.

Em revisão bibliográfica para analisar a acessibilidade e as possíveis dificuldades quanto às barreiras arquitetônicas de pacientes cadeirantes por esclerose 
lateral amiotrófica concluiu-se que estudos sobre acessibilidade em ambiente domiciliar ainda são escassos na literatura científica (SOUZA; PERES, 2007).

Algumas medidas são eficazes para melhorar a acessibilidade e evitar quedas e acidentes; tais como: instalação de corrimãos, aumento da iluminação, instalação de faixas coloridas nos degraus de escadas para identificar mudanças de nível são soluções para prevenir quedas.

Quando analisados os itens acessíveis em uso coletivo pelas pessoas com deficiência física, novamente constatou-se que "rampas de acesso" foi o mais marcado $(35,4 \%)$ seguido de vaga de estacionamento reservada $(32,9 \%)$, transporte público adaptado (26,2\%). No entanto, "barras de apoio no chuveiro" e "torneiras adaptadas" foram apontadas com $6,1 \%$ do total de respondentes como os itens menos acessíveis. Os itens mais acessíveis da população estudada apontam que ainda existem barreiras arquitetônicas na vida das pessoas com deficiência física.

Pode-se observar no presente estudo como a acessibilidade dos espaços de uso coletivo é restrita para as pessoas com deficiência física do município de Itajaí/SC. Corroborando com tal achado de Kovaleski; Baldissera (2015), que ao avaliarem a acessibilidade física nas instituições públicas de outro município Catarinense, também notaram barreiras arquitetônicas tais como: falta de rampas de acessos nos espaços de uso coletivo, calçadas públicas que não são totalmente adaptadas ou não são adaptadas; estacionamentos que não são todos adaptados; não possui piso tátil; os banheiros não são adaptados.

Lamônica et al. (2008), que identificaram e descreveram barreiras arquitetônicas na Universidade de São Paulo (USP) no campus de Bauru, perceberam rampas de acesso com piso tátil no início e no final das rampas, porém tanto nas rampas quanto nas escadas não possuíam corrimões, os banheiros eram parcialmente adaptados, verificaram-se poucas vagas de estacionamento e poucos elevadores, no entanto, após obterem esta análise, realizaram intervenções na universidade e em seguida observaram melhorias.

No estudo de Freitas et al. (2010), foram analisados itens necessários para acesso de portadores de deficiências física e sensorial na Universidade Estadual de Campinas (UNICAMP) em São Paulo. Aonde verificou-se que dos seis espaços avaliados só um apresentou ausência de barreiras arquitetônicas, nos outros se pode notar falta de rampas de acesso nos espaços de uso coletivo, assim como a falta de estacionamento, elevadores, de portas amplas nas salas de aula, além de escadas sem corrimões, banheiros não adaptados e trechos de calçadas estreitas.

No estudo de Castro et al. (2011), que realizou um estudo sobre a acessibilidade aos serviços de saúde por pessoa com deficiência no estado de São Paulo, com uma amostra intencional de 25 indivíduos portadores de deficiência física, encontraram resultados semelhantes ao de nosso estudo e de outros autores citado acima, pois 
parte dos entrevistados relataram falta de estacionamento nesses ambientes, seguido de falta de rampas de acesso e também um critério que chama atenção é a falta de banheiros adaptados a esse público, sendo que a acessibilidade por si só é de total direito do deficiente físico.

Como nos mostra BRASIL (2000), foi dado o decreto 3.298/99 que regulamenta a lei 7.893/89, aonde diz que todo o deficiente físico, dispõe sobre obrigatoriedade a presença de vagas reservadas para seus veículos ou para quem transporte-os, por fim esse decreto dá o direito de ter vagas reservadas aos deficientes físicos. Ainda sobre decreto 3.298/99 aonde o mesmo obriga a instalação de rampas de acesso ou dispositivos eletromecânicos para o deslocamento do deficiente físico para se deslocar de um cômodo a outro nesses ambientes. A lei №10. 098 ainda garante o direito desses cidadãos com deficiência física de ter acesso a banheiros adaptados (BRASIL, 2000).

Pagliuca, Aragão e Almeida (2007), mostram em sua pesquisa sobre a identificação de barreiras arquitetônicas em áreas internas de hospitais de Sobral, Ceará, com relação a acessibilidade e a deficiência física, uma análise de quatro hospitais que identificam e confirmam barreiras arquitetônicas no interior desses hospitais, com irregularidades em altura das rampas de acesso, sendo que algumas não tem barras de apoio bilateralmente para suporte do acesso dos pacientes, fora outros achados que chamam atenção, porém o item de rampas de acesso condiz com o nosso estudo, evidenciando a falta ou a irregularidade do mesmo nesses ambientes que pessoas com deficiência física tem acesso.

Por fim, ressalta-se que examinando dados do presente estudo, bem como dos demais trabalhos encontrados na literatura científica consultada, é possível afirmar que ainda existe a eminente falta de acessibilidade para as pessoas com deficiência em esferas de uso coletivo.

\section{CONCLUSÃO}

O desenvolvimento do presente trabalho possibilitou analisar a percepção sobre alguns aspectos da acessibilidade da pessoa com deficiência física residente no município Itajaí/SC, bem como uma revisão bibliográfica sobre a deficiência física e a acessibilidade arquitetônica.

Por fim, considera-se que na percepção do deficiente físico ainda existem muitas barreiras arquitetônicas no âmbito domiciliar e no coletivo que não os favorecem, impossibilitando o desempenho de locomoção impedindo a pessoa de ter autonomia e assim conseguir exercer plenamente seu direito de "ir e vir" expresso nas legislações vigentes. 


\section{REFERÊNCIAS}

ASSOCIAÇÃO BRASILEIRA DE NORMAS TÉCNICAS (ABNT). NBR 9050.

Acessibilidade a edificações, mobiliário, espaços e equipamentos urbanos. 2. ed. Rio de Janeiro: ABNT, 2004;

Decreto n. 10098, p.1, de 19 de dezembro de 2000. Estabelece normas gerais e critério básicos para a promoção da acessibilidade das pessoas portadoras de deficiência ou com mobilidade reduzida, e dá outras providências. Diário Oficial da União, Brasília, 2000.

CASTRO, Shamyr Sulyvan et al. Acessibilidade aos serviços de saúde por pessoas com deficiência. Rev. Saúde Pública, São Paulo, v. 45, n. 1, p. 99-105, fev. 2011. Disponível em. <http://www.scielo.br/scielo.php?script=sci_arttext\&pid=S0034$89102011000100011 \&$ Ing=en\&nrm=iso >. Acesso em: 01 jun. 2017.

CASTRO, Shamyr Sulyvan de et al. Deficiência visual, auditiva e física: prevalência e fatores associados em estudo de base populacional. Cad. Saúde Pública, Rio de Janeiro, v. 24, n. 8, p. 1773-1782, ago. 2008. Disponível em: <http://www.scielo.br/scielo.php?script=sci_arttext\&pid=S0102311X2008000800006\&lng=en\&nrm=iso>. Acesso em: 01 mar. 2017.

ELIAS, M. P.; MONTEIRO, L. M. C.; CHAVES, C. R. Acessibilidade a benefícios legais disponíveis no Rio de Janeiro para portadores de deficiência física. Ciência \& Saúde Coletiva, Rio de Janeiro, v. 13, n. 3, p.1041-1050, 2008. Disponível em: $<$ http://www.scielo.br/scielo.php?script=sci_arttext\&pid=S1413$81232008000300027 \&$ Ing=en\&nrm=iso $>$. Acesso em: 01 fev 2017.

FARIAS, N.; BUCHALLA, C. M. A Classificação Internacional de Funcionalidade, Incapacidade e Saúde da Organização Mundial da Saúde: Conceitos, usos e perspectivas. Revista Brasileira de Epidemiologia, v. 8, n. 2, p. 187-193, 2005. Disponível em: <http://www.scielo.br/scielo.php?script=sci_arttext\&pid=S1413$81232008000300027 \&$ Ing=en\&nrm=iso>. Acesso em: 05 maio 2017.

FREITAS, A. P. et al. Análise dos itens necessários para acesso de portadores de deficiências físicas e sensoriais na Unicamp. Revista Ciências do Ambiente, v. 6, n. 1, 2010. Disponível em:

<http://sistemas.ib.unicamp.br/be310/nova/index.php/be310/article/viewFile/403/324>. Acesso em: 11 de maio 2017.

GARANHANI, M. R.; ALVES, J. F.; FUJISAWA, D.S. Adaptação da pessoa pósacidente vascular encefálico e seu cuidador: ambiente domiciliar, cadeira de rodas e de banho. In CONGRESSO BRASILEIRO MULTIDISCIPLINAR DE EDUCAÇÃO ESPECIAL, 5, 2009. Anais..., Londrina, PR. 2009.

GASPAROTO, M. C.; ALPINO, Â. M. S. Avaliação da acessibilidade domiciliar de crianças com deficiência física. Revista brasileira de educação especial, Marília, v. 18, n. 2, p. 337-354, jun. 2012. Disponível em <http://www.scielo.br/scielo.php?script=sci_arttext\&pid=S141365382012000200011\&lng=pt\&nrm=iso >. Acesso em: 01 mar. 2017.

INSTITUTO BRASILEIRO DE GEOGRAFIA E ESTATÍSTICA (IBGE). Resultado do Censo Demográfico 2010. Rio de Janeiro: IBGE; 2013. 
KOVALESKI, M. C.; BALDISSERA, A. D. Acessibilidade física nas instituições públicas de Chapecó-SC. Revista CientíficaTecnológica, v. 3, n. 2, p. 34-49, 2015.

LAMÔNICA, D. A. C. et al. Acessibilidade em ambiente universitário: identificação de barreiras arquitetônicas no campus da USP de Bauru. Revista Brasileira de Educação Especial, Marília - SP, v. 14, n. 2, p. 177-188, agosto. 2008. Disponível em: <http://www.producao.usp.br/handle/BDPI/11021>. Acesso em: 15 de abr. 2017.

LEYTON, V. et al. Perfil epidemiológico das vítimas fatais por acidente de trânsito e a relação com o uso do álcool. Saúde, Ética \& Justiça. v. 10, n. 1/2, p. 12-18, 2005.

MACHADO, M. H.; LIMA, J. P. Avaliação multicritério da acessibilidade de pessoas com mobilidade reduzida: um estudo na região central de Itajubá (MG). Revista Brasileira de Gestão Urbana, Itajubá - MG, v. 7, n. 3, p. 368-382, 2015. Disponível em: <http://www.scielo.br/scielo.php?script=sci_arttext\&pid=S217533692015000300368\&lng=en\&nrm=iso>. Acesso em: 01 abr. 2017.

NOGUEIRA, G. C. Perfil epidemiológico da pessoa com deficiência física residente em Florianópolis. 2014. Dissertação (Mestrado em Enfermagem) Universidade Federal de Santa Catarina, Florianópolis, 2014.

PYNOOS, J.; OVERTON, J. A changing environment. Rehab. Management.; v. 16, n. 2, p. 38-42, 2003.

PAGLIUCA, L. M. F; ARAGAO, A. E. A; ALMEIDA, P. C. Acessibilidade e deficiência física: identificação de barreiras arquitetônicas em áreas internas de hospitais de Sobral, Ceará. Rev. esc. enferm., v. 41, n. 4, p. 581-588, 2007. Disponível em: $<$ http://www.scielo.br/scielo.php?script=sci_arttext\&pid=S0080$62342007000400007 \&$ Ing=en\&nrm=iso >. Acesso em: 01 set. 2017.

SCHOELLER, S. D. et al. Knowing to care: characterization find individuals withspinalcordin jury treatedat a rehabilitation center. Fisioterapia em Movimento, Curitiba, v. 28, n. 1, p. 77-83, março, 2015. Disponível em: $<$ http://www.scielo.br/scielo.php?script=sci_arttext\&pid=S0103$51502015000100077 \&$ lng=en\&nrm=iso >. Acesso em 05 fev. 2017.

SOUZA, F. R.; PERES, F. R. Análise da acessibilidade e as possíveis dificuldades quanto às barreiras domiciliar de pacientes cadeirantes por esclerose lateral amiotrófica: uma revisão da literatura. 2007. Tese (Especialização em Intervenção Fisioterapeutica em Doenças Neuromusculares pela Escola Paulista de Medicina) Universidade Federal de São Paulo, UNIFESP, São Paulo, 2007.

STATSOFT, Inc. (2011). STATISTICA (data analysis software system), version 10. Disponível em: <www.statsoft.com>. Acesso em: 13 maio 2016. 